\title{
Derivation of Poly-Methylomic Profile Scores for Schizophrenia
}

Watkeys, Oliver J. ${ }^{\text {a, b }}$

Cohen-Woods, Sarah ${ }^{\mathrm{c}, \mathrm{d}, \mathrm{e}}$

Quidé, Yann ${ }^{\mathrm{a}, \mathrm{b}}$

Cairns, Murray J. ${ }^{\mathrm{f}}$

Bronwyn Overs ${ }^{b}$

Fullerton, Janice M. ${ }^{\text {b, g }}$

Green, Melissa J. * a, b

${ }^{\text {a }}$ School of Psychiatry, University of New South Wales (UNSW Sydney), Sydney, NSW,

Australia

${ }^{\mathrm{b}}$ Neuroscience Research Australia, Sydney, NSW, Australia

${ }^{\mathrm{c}}$ Discipline of Psychology, Flinders University, Adelaide, SA, Australia

${ }^{\mathrm{d}}$ Flinders Centre for Innovation in Cancer, Adelaide, SA, Australia

${ }^{\mathrm{e}}$ Centre for Neuroscience, Adelaide, SA, Australia

${ }^{\mathrm{f}}$ School of Biomedical Sciences and Pharmacy, University of Newcastle, Newcastle, NSW, Australia

${ }^{\mathrm{g}}$ School of Medical Sciences, University of New South Wales (UNSW Sydney), Sydney, NSW, Australia

\section{*Corresponding Author}

Professor Melissa Green, UNSW Research Unit for Schizophrenia Epidemiology, Level 4, The O'Brien Centre, St Vincent's Hospital, 394-404 Victoria Street, Darlinghurst NSW 2010. Telephone: +61 028382 1584. Email: melissa.green@unsw.edu.au

Keywords: Epigenetics, psychosis, polygenic, bipolar, EWAS, methylation

Word count: 3,789

Number of Tables: 4

Number of Figures: 1

Supplementary Materials: 3 Tables 


\begin{abstract}
Schizophrenia (SZ) and bipolar disorder (BD) share numerous clinical and biological features as well as environmental risk factors that may be associated with altered DNA methylation. In this study we sought to construct a Poly-Methylomic Profile Score (PMPS) for SZ, representing the degree of epigenome-wide methylation according to previously published findings; we then examined its association with SZ and BD in an independent sample. DNA methylation for $57 \mathrm{SZ}, 59 \mathrm{BD}$ cases and 55 healthy controls (HCs) was quantified using the Illumina 450K methylation beadchip. We constructed five PMPSs for different $\mathrm{p}$-value thresholds using summary statistics reported in a large epigenome-wide schizophrenia casecontrol association study, weighted by individual CpG effect sizes. All SZ PMPSs were significantly elevated in SZ cases relative to HCs, with the score calculated at the most stringent threshold accounting for the greatest amount of variance in SZ (compared to other PMPSs derived at more inclusive $p$-value thresholds). However, none of the PMPSs were associated with $\mathrm{BD}$, or a combined cohort of $\mathrm{BD}$ and $\mathrm{SZ}$ cases relative to HCs. Results demonstrating elevated PMPSs in SZ relative to BD did not survive correction for multiple testing. PMPSs were also not associated with positive or negative symptom severity. That this SZ-derived PMPSs was elevated among SZ, but not BD participants, suggests that epigenome-wide methylation patterns associated with schizophrenia may represent distinct pathophysiology that is yet to be elucidated. Whether this PMPS may be associated with neuroanatomical or other biological endophenotypes relevant to $\mathrm{SZ}$ and/or BD remains to be determined.
\end{abstract}




\section{Introduction}

Owing to recent advances in genomic technologies and collaboration among global consortia, schizophrenia (SZ) and bipolar disorder (BD) have been shown to share a significant proportion of genetic liability (Hamshere et al., 2011; Ivleva et al., 2010; Purcell et al., 2009). For example, the degree to which multiple sites of genetic variation contribute to risk for $\mathrm{SZ}$ and $\mathrm{BD}$ can be summarised using 'polygenic risk scores', calculated as the sum of alleles associated with a particular trait, weighted by their respective effect sizes (Purcell et al., 2009). While the majority of these genetic variants would individually fail to reach even nominal levels of statistical significance, collectively they may account for a significant proportion of the variance in manifestation of SZ and BD (Purcell et al., 2009). However, there remains a significant amount of unexplained variance in shared genetic and environmental risk for these disorders (Lichtenstein et al., 2009), some of which may be accounted for by epigenetic processes associated with shared environmental risk factors such as childhood trauma, cannabis use, obstetric complications (Bourque et al., 2011; Buoli et al., 2016; Cannon et al., 2002; Kraan et al., 2016; Laursen et al., 2007).

Epigenetic processes, such as DNA methylation, refer to functionally significant alterations in the genome that do not alter the nucleotide sequence (Hannon et al., 2016; Montano et al., 2016; Pries et al., 2017). DNA methylation involves the addition of a methylgroup to the carbon-5 position of a cytosine residue (Razin and Cedar, 1991): this may be associated with increased or decreased gene expression depending on the genomic region in which it occurs (Jones, 2012; Watkeys et al., 2018). Both candidate- and genome-wide studies have revealed differential methylation of various sites in SZ (Hannon et al., 2016; Montano et al., 2016; Ruzicka et al., 2015; Yoshino et al., 2016), with some studies suggesting overlapping patterns of DNA methylation in BD (Dempster et al., 2011; Sugawara 
et al., 2018), although there have been few replications at individual CpG sites (Pries et al., 2017; Teroganova et al., 2016).

A recently published epigenome-wide association study (EWAS) (Hannon et al., 2016) of blood-derived DNA methylation patterns reported 14 differentially methylated CpG sites that were robustly associated with SZ in both a large discovery sample (comprising 353 SZ cases and 322 healthy controls) and a replication sample (comprising 414 SZ cases and 433 healthy controls), at a stringent significance level $\left(p<1 \times 10^{-7}\right)$. A larger number of CpGs $(n=213)$ were associated with SZ in both the discovery and replication case-control samples at a more liberal significance threshold $\left(p<1 \times 10^{-5}\right)$. Hannon et al.'s gene ontology analysis suggested that genes containing CpG sites associated with SZ were implicated in inflammatory processes and brain development. Importantly, Hannon et al. (Hannon et al., 2016) reported effect sizes and $p$-values for all $\mathrm{CpG}$ sites differentially methylated in SZ for all samples (with replication $p$-values ranging from $4.42 \times 10^{-18}$ to 0.991 ), and included covariates (age, sex, technical batch effects, as well as methylation-derived estimates of cell composition and smoking status). A comparably sized study published in the same year reported only $\mathrm{CpG}$ sites significant at an FDR corrected threshold of $p<0.2$ in their replication sample (number CpGs = 172) (Montano et al., 2016).

In this study, we adapted methodology used to calculate PRS from GWAS summary statistics, to estimate a series of poly-methylomic profile scores (PMPSs) for SZ. The calculation of these scores was based on discovery effect sizes of individual differentially methylated $\mathrm{CpG}$ sites at various $p$-value thresholds as reported by Hannon et al. (2016). We then established the extent to which these PMPSs were associated with an independent sample of cases with SZ, BD, and the combined SZ-BD group (each compared to healthy controls; HC), and directly compared PMPSs between SZ and BD. We also explored 
associations with positive and negative symptoms of psychosis for the PMPS that was most strongly associated with SZ.

\section{Experimental/Materials and methods.}

Study procedures were approved by the UNSW Human Research Ethics committees (HC12384), St. Vincent's Hospital (HREC/10/SVH/9), and the South East Sydney and Illawarra Area Health Service (HREC 09/081).

\subsection{Participants}

Peripheral blood samples were obtained from a total of 191 study participants (64 SZ, $64 \mathrm{BD}, 60 \mathrm{HC}$ ). Following the application of exclusion criteria and QC processes (see below) the final sample $(\mathrm{N}=171)$ comprised 57 people who met ICD-10 (World Health Organisation, 2004) criteria for SZ or schizoaffective disorder (referred to as SZ), 59 individuals with BD-I disorder (BD), and 55 HCs. Clinical participants were recruited from outpatient services of the South Eastern Sydney-Illawarra Area Health Service (SESIAHS), the Australian Schizophrenia Research Bank (Loughland et al., 2010), and the Sydney BD Clinic (Mitchell et al., 2009). Healthy controls were recruited from the local community. Study exclusion criteria included current neurological disorder, current substance abuse or dependence, and/or electroconvulsive treatment in the past six months. Healthy controls were also required to have no lifetime history of a DSM-IV Axis-I diagnosis (American Psychiatric Association, 1994) confirmed using the Mini-International Neuropsychiatric Interview (Sheehan et al., 1998), and no history of psychotic disorders among first-degree biological relatives.

\subsection{Cognitive and symptom measures}

Estimates of premorbid and current intelligence quotients (IQ) were calculated using the Wechsler Test of Adult Reading (Wechsler, 2001), and the Wechsler Abbreviated Scale of Intelligence (Wechsler, 1999), respectively. The Scales for the Assessment of Positive 
Symptoms (SAPS) and Negative Symptoms (SANS) were used to assess symptom severity (Andreasen, 1984a, b).

\subsection{Methylation quantification and quality control}

Methylation quantification was undertaken on DNA samples from 191 participants at the Institute for Molecular Bioscience, University of Queensland (UQ), according to standard manufacturer's instructions using the Illumina 450K BeadChip. Samples were randomised on the plate to avoid bias due to chip or position on chip. Quantification was completed using the Qubit fluorimeter, and bisulfite conversions were completed using the EZ-96 DNA Methylation kit according to the manufacturers protocol (Zymo Research, Orange, CA, USA), and assessed on the Epoch Microplate Spectrophotometer (Biotek, Winooski, VT). All chips were imaged and analysed for DNA methylation status on the Illumina iScan system using the GenomeStudio methylation module software (Illumina, San Diego, CA, USA). This produced a methylation score as a continuous $\beta$-value representing the ratio of methylated (M) probe intensity relative to total intensity of methylated and unmethylated (U) probes defined as $\beta=M /(M+U)$. Raw and total probe intensities were extracted.

Of the 191 samples, 94 had DNA derived from whole blood, 94 firstly underwent ficoll density gradient separation with DNA extracted from the cells in the ficoll/PBS/plasma layer, and 3 were derived from the isolated lymphoblast cell population after ficoll separation; each with different cellular compositions in each tissue source. Ten samples were excluded from methylation QC analyses owing to their lymphoblast origin (3 samples) or having undergone 450k hybridisation in a different batch (7 samples); 181 DNA samples (whole blood or ficoll-treated) were included in methylation QC procedures.

The R package 'meffil' (Min et al., 2017) was used to extract signal intensities and perform background correction and normalisation of methylation beta values for individual CpG sites. Our QC procedure involved excluding individual subjects for whom: (i) more than 
$10 \%$ of probes had less than three beads ( 0 samples), (ii) the predicted median methylation signal was in excess of three standard deviations from the regression line generated from methylated vs. unmethylated signal intensities (1 sample), (iii) a gender mismatch (0 samples) or XY outlier status was detected (2 samples), (iv) methylation signal intensities deviated excessively from mean values for control probes (4 samples), (v) more than $10 \%$ of probes had a detection $p$-value $>0.01$ ( 0 samples), or (vi) a genotype mismatch with genotypes derived from Illumina psych-chip was detected (1 sample). Individual probes were excluded if (i) $>10 \%$ of samples showed detection $p$-values $>0.01$ for that probe $(n=137)$, (ii) $>10 \%$ of samples demonstrated less than 3 beads at a given probe $(n=141)$, or (iii) the probe was previously demonstrated to be cross-reactive $(n=29233)$ (Chen et al., 2013). After application of these QC procedures, $456041 \mathrm{CpG}$ sites remained for analysis in the 171 participants. However, in the current study we only included the 1051 independent CpG sites demonstrating a replication $p$-value $<0.5$ in Hannon et al.'s (Hannon et al., 2016) first replication cohort.

Blood cell count estimates were derived from methylation data for Bcells, CD4T, CD8T, granulocytes, monocytes, and natural killer cells using meffil's gse35069 profile references. Shinymethyl (Fortin et al., 2014) was used to conduct principle components analysis with the final QCed data. We found two components significantly associated with ethnicity $(p<0.05)$, which were included as covariates in all subsequent analysis.

\subsection{Poly-Methylomic Profile Score (PMPS)}

PMPSs were calculated utilising data from multiple $\mathrm{CpG}$ sites previously reported as demonstrating an association with SZ (Hannon et al., 2016), weighted on the basis of discovery sample effect sizes. Sites were included in the PMPSs if they replicated across the two cohorts tested by Hannon et al. (2016) at five different $p$-value thresholds $\left(1 \times 10^{-7}, 1 \times 10^{-}\right.$ $\left.{ }^{5}, 0.001,0.05,0.5\right)$. The use of multiple $p$-value thresholds is standard in the construction of 
polygenic risk scores, in order to identify the threshold that enables optimal prediction (Purcell et al., 2009). The five $p$-value thresholds included 14, 210, 359, 684, and $1051 \mathrm{CpG}$ sites in each PMPS, respectively. The first two scores were calculated based on CpG sites which replicated at their respective $p$-value threshold across both the discovery and first replication cohort. In contrast, the final three PMPSs were calculated based on sites that evidenced at least a discovery threshold $\left(p<1 \times 10^{-5}\right)$ association with schizophrenia and replicated at each of the three thresholds listed above, respectively (Hannon et al., 2016). Each PMPS score was calculated using previously described methods (Shah et al., 2015) as the sum of weighted beta values associated with SZ, or:

$$
P M P S i=\sum B i j x \Delta j
$$

Where PMPSi is the polymethylomic profile score for the $\mathrm{i}^{\text {th }}$ participant, $\mathrm{Bij}$ is the beta value for the $\mathrm{i}^{\text {th }}$ participant at the $\mathrm{j}^{\text {th }} \mathrm{CpG}$ site, and $\Delta \mathrm{j}$ is the effect size for the $\mathrm{j}^{\text {th }} \mathrm{CpG}$ site.

To ensure that each PMPS was not inflated by correlated methylation among CpGs, the independence of $\mathrm{CpG}$ sites meeting thresholds for inclusion in any PMPS was assessed via correlation analyses. In the event that two or more $\mathrm{CpG}$ sites within 500 base-pairs (bp) of each other demonstrated a Pearson's correlation coefficient $>0.1$, a single site demonstrating the strongest association with SZ (as indicated by replication $p$-values from Hannon et al. (2016) was retained for PMPS calculation, whilst the other correlated sites $(n=10)$ were excluded (see Supplementary Table 1) (Huynh et al., 2013; Ong and Holbrook, 2013; Shah et al., 2015).

As smoking behaviour influences methylation (Zeilinger et al., 2013), we used the method described above to estimate a methylation smoking score, using smoking-related methylation effect sizes for $\mathrm{CpG}$ sites demonstrating a significant replicated association with smoking status ( $p$-value $<5 \times 10^{-5}$ ) reported previously (Zeilinger et al., 2013). We validated this score using smoking data collected for our clinical participants; the smoking score was 
significantly associated with both lifetime smoking $\left(B=0.880, O R=2.410, p=6.49 \times 10^{-5}\right)$ and Fagerstrom test for nicotine dependency (Heatherton et al., 1991) $(B=0.395, O R=$ $1.484, p=0.004)$.

\subsection{Statistical Analyses}

An initial exploratory analysis investigated whether patterns of methylation for individual CpG sites reported by Hannon et al. (2016) were differentially methylated in the current SZ sample. Beta values for each $\mathrm{CpG}$ site were used as dependent variables and clinical status $(\mathrm{SZ} / \mathrm{HC})$ as the main explanatory variable. For this and all subsequent analyses, covariates of age, sex, tissue source (ficoll or whole blood), estimated methylation-derived cell counts, two methylation-derived ethnicity principle components, and an estimated methylation smoking score were included as fixed factors, and 450k slide ID as a random factor. Individual $\mathrm{CpG}$ sites were only considered to be significantly associated with $\mathrm{SZ}$ if they evidenced a False Discovery Rate (FDR) corrected $q$-value $<0.05$.

A series of logistic regression analyses were then performed to assess if the PMPSs were associated with: (i) SZ (relative to $\mathrm{HC}$ ); (ii) BD (relative to $\mathrm{HC}$ ); (iii) the combined BD and SZ group (relative to $\mathrm{HC}$ ); and (iv) SZ (relative to BD). The PMPSs were converted to zscores before analysis so that regression coefficients and effect sizes would be comparable between models. FDR correction was applied across the five PMPSs assessed within each group comparison. In addition, two linear regression analyses were used to assess if the optimal PMPS was associated with SAPS and SANS global subscale scores respectively.

To investigate any potential confounding effects of medication, we ran three linear regression models (one model each for $\mathrm{BD}, \mathrm{SZ}$, and the combined SZ-BD cohort), with the PMPS that explained the most variance in SZ as the dependent variable, and imipramine (antidepressant) equivalency dosage, chlorpromazine (antipsychotic) equivalency dosage, and 
mood stabiliser usage included as predictors in addition to covariates used in all other analyses.

\section{Results}

Demographic information for included participants is summarised in Table 1.

\subsection{Differential methylation analysis}

Of $1051 \mathrm{CpG}$ sites included for analysis in the present study, only 79 were associated with SZ in our sample at a nominal $p$-value threshold $(p<0.05)$, with none surviving FDR correction for multiple testing (FDR $<0.05$; see Supplementary Tables 2 and 3 for full results). Of the $14 \mathrm{CpG}$ sites most robustly associated with SZ in both Hannon et al.'s (2016) discovery and replication cohorts $\left(p<1 \times 10^{-7}\right)$, only two sites ( $\operatorname{cg} 13803727$ and $\left.\operatorname{cg} 27541604\right)$ demonstrated nominally significant associations with SZ in our cohort $(p<0.05)$ (see Supplementary Table 2). There was 100\% consistency at these 14 sites between the direction of hypo-/hyper-methylation reported in Hannon et al.'s (Hannon et al., 2016) discovery cohort and our own sample. The consistency in the direction of methylation between Hannon et al. (2016) discovery cohort and our own $(81.42 \%, 74.93 \%, 64.91 \%$, and $58.23 \%)$ tended to decrease at more liberal $\mathrm{p}$-value thresholds $\left(1 \times 10^{-5}, 0.001,0.05\right.$, and 0.5 , respectively).

\subsection{PMPS model comparisons in association with SZ}

All five PMPSs were significantly associated with SZ relative to HC both before and after FDR correction (see Table 2). All models explained a similar amount of the variance in SZ (58-60\%), however models incorporating PMPSs based on more liberal $p$-value inclusion thresholds tended to explain less of the variance (see Table 2). The PMPS that explained the greatest amount of variance in $\mathrm{SZ}$ diagnosis incorporated the $14 \mathrm{CpG}$ sites which were those most robustly associated with SZ across both discovery and replication samples from Hannon et al. (2016) (Nagelkerke Pseudo $\left.\mathrm{R}^{2}=0.595\right)$. Within this model, SZ cases showed 
heightened PMPS relative to controls $(B=1.263, O R=3.538,95 \% \mathrm{CI}=1.227-10.197, p=$ 0.018; see Figure 1).

\subsection{SZ-PMPS in association with BD and the cross-disorder sample}

In contrast to the SZ-HC PMPS models (described above), models comparing BD and the combined "psychosis spectrum" group ( $\mathrm{SZ}+\mathrm{BD}=116$ cases) to HCs, explained considerably less of the variance (39-41\% and $31-33 \%$, respectively). Furthermore, none of the SZ PMPSs within these models were significantly associated with a diagnosis of BD (relative to HCs), nor were they significantly associated with the combined "psychosis spectrum" group relative to HCs either before or after FDR correction. Conversely, a considerable degree of the variance in BD versus SZ status was accounted for by the PMPS models (51-53\%). While there was no significant difference between BD and SZ participants on the PMPS calculated at the most stringent $p$-value threshold $\left(p<1 \times 10^{-7}\right)(B=0.852, O R$ $=2.345,95 \% \mathrm{CI}=0.970-5.666, p=0.055)$ all other PMPS scores were significantly elevated in $\mathrm{SZ}$ relative to $\mathrm{BD}$. However, none of these associations withstood FDR correction (see Table 2).

3.4. Associations between optimal PMPS and positive and negative symptoms of psychosis

There were no relationships between the optimal PMPS and any subscales of the SAPS and SANS, respectively (see Table 3).

\subsection{Potential effects of medication on PMPS scores}

Irrespective of clinical group (SZ, BD, or cross-diagnostic "psychosis spectrum" group), all linear regression analyses indicated that medication usage was not significantly associated with the optimal PMPS (all $p>0.05$; see Table 4).

\section{Discussion}

Here we demonstrate the construction of a Poly-Methylomic Profile Score for schizophrenia based on differentially methylated CpG sites reported in a recent EWAS in 
schizophrenia (2016); we demonstrate significantly higher scores in an independent schizophrenia sample, relative to healthy controls, at both stringent and increasingly liberal thresholds of significance. Although we found little evidence for significant differences in methylation at those individual $\mathrm{CpG}$ sites in this small schizophrenia sample, likely due to limited power, there was $100 \%$ consistency in the direction of DNA hyper-/hypo-methylation in our schizophrenia sample among the $14 \mathrm{CpG}$ sites previously reported as most strongly associated with schizophrenia (Hannon et al., 2016). Interestingly, schizophrenia PMPSs were not associated with BD or the combined psychosis group compared to healthy controls, suggesting that the SZ-derived PMPS may reflect distinct pathophysiology in SZ.

Furthermore, whilst the PMPS differences between SZ and BD were not significant after FDR correction, these associations were only just above the threshold of statistical significance. Whilst it is possible that this indicates shared methylation patterns between SZ and $\mathrm{BD}$, it is also possible that our study was underpowered to detect true differences in the methylation profiles between SZ and BD.

The capacity to demonstrate associations between the constructed poly-methylomic scores and schizophrenia is consistent with the idea that methylation changes in schizophrenia span multiple $\mathrm{CpG}$ sites of small effect throughout the genome, and that collectively, the summed effects across multiple $\mathrm{CpG}$ sites should explain a larger proportion of the variance in the manifestation of schizophrenia than individual $\mathrm{CpG}$ sites alone. Notably, the amount of variance explained by the PMPSs decreased as increasingly liberal $p$ value thresholds were adopted for score calculation. This stands in stark contrast to genetic polygenic risk scores, which tend to explain more of the variance when more liberal $p$-value thresholds are utilised (Purcell et al., 2009). This difference may reflect the smaller EWAS cohorts compared to those used to generate polygenic risk scores for schizophrenia (Purcell et al., 2009). However, additional consideration should also be given to the increased extent to 
which DNA methylation is susceptible to confounding factors. That is, whilst genetic sequence variants are inherited directly from parents and remain stable across the lifespan (except in the case of de novo events), epigenetic processes are susceptible to environmental modification and change over time (Dempster et al., 2013). These factors make it inappropriate to characterise a trait-associated methylation score as indexing risk for the trait in question. Rather, any such score might be instead regarded as summarising the methylation profile associated with a given trait. Indeed, such scores have already been calculated for phenotypes such as body-mass index (BMI) and environmental exposures such as smoking status, education, and alcohol consumption (Elliott et al., 2014; Hamilton et al., 2018; McCartney et al., 2018). Our study reflects the first attempt to derive such scores from methylome-wide $\mathrm{CpG}$ data for established schizophrenia. Application of this profile to firstepisode psychosis samples, and the further derivation of PMPSs from EWASs conducted in first-episode psychosis and other medication naive high-risk samples, will be useful to understand its potential biological relevance to disease risk and the emerging pathophysiology of schizophrenia.

Interestingly, none of the SZ-derived PMPS scores were associated with BD or the cross-disorder psychosis group relative to $\mathrm{HCs}$, suggesting that the methylation patterns summarised by these scores may reflect distinct schizophrenia pathophysiology. This was somewhat unexpected given increasing evidence of biological overlap between schizophrenia and BD (Dempster et al., 2011; Ivleva et al., 2010; Laursen et al., 2007; Purcell et al., 2009; Sugawara et al., 2018). Furthermore, whilst PMPSs were not significantly elevated in SZ compared to BD after correcting for multiple testing, this could reflect lack of power. Larger discovery EWASs may be required to identify CpGs that are differentially methylated in both conditions. It would also be interesting to determine if a PMPS calculated using discovery 
data from a BD cohort is associated with both $\mathrm{BD}$ and SZ, however large discovery EWAS datasets in bipolar disorder are currently lacking.

Several limitations should be considered when interpreting these results. First, the reference profile used for estimating cellular composition was based on a small sample of men (Reinius et al., 2012). Whilst this method is widely used, questions remain regarding the validity of this measure as a means of estimating cellular composition in the broader population. Other potential sources of variation in DNA methylation include participants' smoking behaviours (Zeilinger et al., 2013) and body mass index (BMI) (McCartney et al., 2018). We partially addressed this by estimating a methylation-based smoking score for use as a covariate (Elliott et al., 2014), since smoking status can be accurately estimated using methylation profile scores (Elliott et al., 2014). However, a methylation-derived score of BMI was not estimated, as there is substantially less reliability where BMI estimation is concerned (McCartney et al., 2018), and phenotypic BMI data was not available for our sample. Regarding potential medication effects, we saw no significant associations between PMPS for schizophrenia and antipsychotic and antidepressant dosages, or mood stabiliser use, but caution that further research in medication-naïve cohorts is warranted. It is also important to note that the tissue specificity inherent to DNA methylation patterns (Davies et al., 2012; Murphy et al., 2005; van den Oord et al., 2016; Walton et al., 2016; Xin et al., 2010) which are highly influenced by cellular composition (Jaffe and Irizarry, 2014), limits the generalisability of methylation patterns obtained from blood. Replicating the methods described here using discovery/replication cohorts derived from brain tissue will be important to understand the significance of methylation patterns over the course of illness.

In conclusion, we have developed a novel method for calculating a poly-methylomic profile of schizophrenia based on published EWAS data; all profile scores derived at various $p$-value thresholds were associated with SZ, but not with BD, or the cross-disorder sample of 
SZ and BD cases, relative to healthy participants. The PMPS has potential utility for estimating how epigenetic patterns across multiple differentially methylated sites might be related to intermediate (endo)phenotypes of schizophrenia and related disorders, such as cognitive functioning and brain structural and functional abnormalities. Future studies in larger independent samples should be mindful of potential demographic and experimental confounders of DNA methylation, such as tissue source of DNA, cellular composition, smoking status, BMI, age and medication exposure. Finally, efforts should be made to assess the extent to which blood-based PMPSs for schizophrenia index methylation patterns in the brain, and the extent to which these markers can shed light on the pathophysiology of psychosis and related disorders. 


\section{References}

American Psychiatric Association, 1994. Diagnostic and statistical manual of mental disorders : DSM-IV. American Psychiatric Association, Washington, DC.

Andreasen, N.C., 1984a. Scale for the Assessment of Negative Symptoms (SANS). University of Iowa, Iowa City, IA.

Andreasen, N.C., 1984b. Scale for the Assessment of Positive Symptoms (SAPS). University of Iowa, Iowa City, IA.

Bourque, F., van der Ven, E., Malla, A., 2011. A meta-analysis of the risk for psychotic disorders among first- and second-generation immigrants. Psychological medicine 41(5), 897-910.

Buoli, M., Bertino, V., Caldiroli, A., Dobrea, C., Serati, M., Ciappolino, V., Altamura, A.C., 2016. Are obstetrical complications really involved in the etiology and course of schizophrenia and mood disorders? Psychiatry Research 241, 297-301.

Cannon, M., Jones, P.B., Murray, R.M., 2002. Obstetric complications and schizophrenia: historical and meta-analytic review. The American journal of psychiatry 159(7), 1080-1092.

Chen, Y.-a., Lemire, M., Choufani, S., Butcher, D.T., Grafodatskaya, D., Zanke, B.W., Gallinger, S., Hudson, T.J., Weksberg, R., 2013. Discovery of cross-reactive probes and polymorphic CpGs in the Illumina Infinium HumanMethylation450 microarray. Epigenetics 8(2), 203-209.

Davies, M.N., Volta, M., Pidsley, R., Lunnon, K., Dixit, A., Lovestone, S., Coarfa, C., Harris, R.A., Milosavljevic, A., Troakes, C., Al-Sarraj, S., Dobson, R., Schalkwyk, L.C., Mill, J., 2012. Functional annotation of the human brain methylome identifies tissuespecific epigenetic variation across brain and blood. Genome Biology 13(6), R43.

Dempster, E.L., Pidsley, R., Schalkwyk, L.C., Owens, S., Georgiades, A., Kane, F., Kalidindi, S., Picchioni, M., Kravariti, E., Toulopoulou, T., Murray, R.M., Mill, J., 2011. Disease-associated epigenetic changes in monozygotic twins discordant for schizophrenia and bipolar disorder. Human Molecular Genetics 20(24), 4786-4796.

Dempster, E.L., Viana, J., Pidsley, R., Mill, J., 2013. Epigenetic studies of schizophrenia: progress, predicaments, and promises for the future. Schizophrenia bulletin 39(1), 11 16.

Elliott, H.R., Tillin, T., McArdle, W.L., Ho, K., Duggirala, A., Frayling, T.M., Davey Smith, G., Hughes, A.D., Chaturvedi, N., Relton, C.L., 2014. Differences in smoking associated DNA methylation patterns in South Asians and Europeans. Clinical epigenetics 6(1), 4.

Fortin, J.P., Fertig, E., Hansen, K., 2014. shinyMethyl: interactive quality control of Illumina 450k DNA methylation arrays in R. F1000Research 3, 175.

Hamilton, O.K.L., Zhang, Q., McRae, A.F., Walker, R.M., Morris, S.W., Redmond, P., Campbell, A., Murray, A.D., Porteous, D.J., Evans, K.L., McIntosh, A.M., Deary, I.J., Marioni, R.E., 2018. An epigenetic score for BMI based on DNA methylation correlates with poor physical health and major disease in the Lothian Birth Cohort 1936. bioRxiv.

Hamshere, M.L., O'Donovan, M.C., Jones, I.R., Jones, L., Kirov, G., Green, E.K., Moskvina, V., Grozeva, D., Bass, N., McQuillin, A., Gurling, H., St Clair, D., Young, A.H., Ferrier, I.N., Farmer, A., McGuffin, P., Sklar, P., Purcell, S., Holmans, P.A., Owen, M.J., Craddock, N., 2011. Polygenic dissection of the bipolar phenotype. The British journal of psychiatry : the journal of mental science 198(4), 284-288. 
Hannon, E., Dempster, E., Viana, J., Burrage, J., Smith, A.R., Macdonald, R., St Clair, D., Mustard, C., Breen, G., Therman, S., Kaprio, J., Toulopoulou, T., Pol, H.E.H., Bohlken, M.M., Kahn, R.S., Nenadic, I., Hultman, C.M., Murray, R.M., Collier, D.A., Bass, N., Gurling, H., McQuillin, A., Schalkwyk, L., Mill, J., 2016. An integrated genetic-epigenetic analysis of schizophrenia: evidence for co-localization of genetic associations and differential DNA methylation. Genome Biology 17(1), 176.

Heatherton, T.F., Kozlowski, L.T., Frecker, R.C., Fagerstrom, K.O., 1991. The Fagerstrom Test for Nicotine Dependence: a revision of the Fagerstrom Tolerance Questionnaire. British journal of addiction 86(9), 1119-1127.

Huynh, J.L., Garg, P., Thin, T.H., Yoo, S., Dutta, R., Trapp, B.D., Haroutunian, V., Zhu, J., Donovan, M.J., Sharp, A.J., Casaccia, P., 2013. Epigenome-wide differences in pathology-free regions of multiple sclerosis-affected brains. Nature Neuroscience 17, 121.

Ivleva, E.I., Morris, D.W., Moates, A.F., Suppes, T., Thaker, G.K., Tamminga, C.A., 2010. Genetics and intermediate phenotypes of the schizophrenia--bipolar disorder boundary. Neuroscience and biobehavioral reviews 34(6), 897-921.

Jaffe, A.E., Irizarry, R.A., 2014. Accounting for cellular heterogeneity is critical in epigenome-wide association studies. Genome Biology 15(2), R31.

Jones, P.A., 2012. Functions of DNA methylation: islands, start sites, gene bodies and beyond. Nature reviews. Genetics 13(7), 484-492.

Kraan, T., Velthorst, E., Koenders, L., Zwaart, K., Ising, H.K., van den Berg, D., de Haan, L., van der Gaag, M., 2016. Cannabis use and transition to psychosis in individuals at ultra-high risk: review and meta-analysis. Psychological medicine 46(4), 673-681.

Laursen, T.M., Munk-Olsen, T., Nordentoft, M., Bo Mortensen, P., 2007. A comparison of selected risk factors for unipolar depressive disorder, bipolar affective disorder, schizoaffective disorder, and schizophrenia from a danish population-based cohort. The Journal of clinical psychiatry 68(11), 1673-1681.

Lichtenstein, P., Yip, B.H., Bjork, C., Pawitan, Y., Cannon, T.D., Sullivan, P.F., Hultman, C.M., 2009. Common genetic determinants of schizophrenia and bipolar disorder in Swedish families: a population-based study. Lancet (London, England) 373(9659), 234-239.

Loughland, C., Draganic, D., McCabe, K., Richards, J., Nasir, A., Allen, J., Catts, S., Jablensky, A., Henskens, F., Michie, P., Mowry, B., Pantelis, C., Schall, U., Scott, R., Tooney, P., Carr, V., 2010. Australian Schizophrenia Research Bank: a database of comprehensive clinical, endophenotypic and genetic data for aetiological studies of schizophrenia. The Australian and New Zealand journal of psychiatry 44(11), 10291035.

McCartney, D.L., Stevenson, A.J., Ritchie, S.J., Walker, R.M., Zhang, Q., Morris, S.W., Campbell, A., Murray, A.D., Whalley, H.C., Gale, C.R., Porteous, D.J., Haley, C.S., McRae, A.F., Wray, N.R., Visscher, P.M., McIntosh, A.M., Evans, K.L., Deary, I.J., Marioni, R.E., 2018. Epigenetic prediction of complex traits and death. bioRxiv.

Min, J., Hemani, G., Davey Smith, G., Relton, C.L., Suderman, M., 2017. Meffil: efficient normalisation and analysis of very large DNA methylation samples. bioRxiv.

Mitchell, P.B., Johnston, A.K., Corry, J., Ball, J.R., Malhi, G.S., 2009. Characteristics of bipolar disorder in an Australian specialist outpatient clinic: comparison across large datasets. The Australian and New Zealand journal of psychiatry 43(2), 109-117.

Montano, C., Taub, M.A., Jaffe, A., Briem, E., Feinberg, J.I., Trygvadottir, R., Idrizi, A., Runarsson, A., Berndsen, B., Gur, R.C., Moore, T.M., Perry, R.T., Fugman, D., Sabunciyan, S., Yolken, R.H., Hyde, T.M., Kleinman, J.E., Sobell, J.L., Pato, C.N., Pato, M.T., Go, R.C., Nimgaonkar, V., Weinberger, D.R., Braff, D., Gur, R.E., Fallin, 
M.D., Feinberg, A.P., 2016. Association of DNA Methylation Differences With Schizophrenia in an Epigenome-Wide Association Study. JAMA psychiatry 73(5), 506-514.

Murphy, B.C., O'Reilly, R.L., Singh, S.M., 2005. Site-specific cytosine methylation in SCOMT promoter in 31 brain regions with implications for studies involving schizophrenia. American journal of medical genetics. Part B, Neuropsychiatric genetics : the official publication of the International Society of Psychiatric Genetics $133 b(1), 37-42$.

Ong, M.-L., Holbrook, J.D., 2013. Novel region discovery method for Infinium 450K DNA methylation data reveals changes associated with aging in muscle and neuronal pathways. Aging Cell 13(1), 142-155.

Pries, L.K., Guloksuz, S., Kenis, G., 2017. DNA Methylation in Schizophrenia. Advances in experimental medicine and biology 978, 211-236.

Purcell, S.M., Wray, N.R., Stone, J.L., Visscher, P.M., O'Donovan, M.C., Sullivan, P.F., Sklar, P., 2009. Common polygenic variation contributes to risk of schizophrenia and bipolar disorder. Nature 460(7256), 748-752.

Razin, A., Cedar, H., 1991. DNA methylation and gene expression. Microbiological reviews $55(3), 451-458$.

Reinius, L.E., Acevedo, N., Joerink, M., Pershagen, G., Dahlén, S.-E., Greco, D., Söderhäll, C., Scheynius, A., Kere, J., 2012. Differential DNA Methylation in Purified Human Blood Cells: Implications for Cell Lineage and Studies on Disease Susceptibility. PLoS ONE 7(7), e41361.

Ruzicka, W.B., Subburaju, S., Benes, F.M., 2015. Circuit- and Diagnosis-Specific DNA Methylation Changes at gamma-Aminobutyric Acid-Related Genes in Postmortem Human Hippocampus in Schizophrenia and Bipolar Disorder. JAMA psychiatry 72(6), 541-551.

Shah, S., Bonder, M.J., Marioni, R.E., Zhu, Z., McRae, A.F., Zhernakova, A., Harris, S.E., Liewald, D., Henders, A.K., Mendelson, M.M., Liu, C., Joehanes, R., Liang, L., Levy, D., Martin, N.G., Starr, J.M., Wijmenga, C., Wray, N.R., Yang, J., Montgomery, G.W., Franke, L., Deary, I.J., Visscher, P.M., 2015. Improving Phenotypic Prediction by Combining Genetic and Epigenetic Associations. American journal of human genetics 97(1), 75-85.

Sheehan, D.V., Lecrubier, Y., Sheehan, K.H., Amorim, P., Janavs, J., Weiller, E., Hergueta, T., Baker, R., Dunbar, G.C., 1998. The Mini-International Neuropsychiatric Interview (M.I.N.I.): the development and validation of a structured diagnostic psychiatric interview for DSM-IV and ICD-10. The Journal of clinical psychiatry 59 Suppl 20, 22-33;quiz 34-57.

Sugawara, H., Murata, Y., Ikegame, T., Sawamura, R., Shimanaga, S., Takeoka, Y., Saito, T., Ikeda, M., Yoshikawa, A., Nishimura, F., Kawamura, Y., Kakiuchi, C., Sasaki, T., Iwata, N., Hashimoto, M., Kasai, K., Kato, T., Bundo, M., Iwamoto, K., 2018. DNA methylation analyses of the candidate genes identified by a methylome-wide association study revealed common epigenetic alterations in schizophrenia and bipolar disorder. Psychiatry and clinical neurosciences 72(4), 245-254.

Teroganova, N., Girshkin, L., Suter, C.M., Green, M.J., 2016. DNA methylation in peripheral tissue of schizophrenia and bipolar disorder: a systematic review. BMC Genetics 17, 27.

van den Oord, E.J., Clark, S.L., Xie, L.Y., Shabalin, A.A., Dozmorov, M.G., Kumar, G., Vladimirov, V.I., Magnusson, P.K., Aberg, K.A., 2016. A Whole Methylome CpGSNP Association Study of Psychosis in Blood and Brain Tissue. Schizophrenia bulletin 42(4), 1018-1026. 
Walton, E., Hass, J., Liu, J., Roffman, J.L., Bernardoni, F., Roessner, V., Kirsch, M., Schackert, G., Calhoun, V., Ehrlich, S., 2016. Correspondence of DNA Methylation Between Blood and Brain Tissue and Its Application to Schizophrenia Research. Schizophrenia Bulletin 42(2), 406-414.

Watkeys, O.J., Kremerskothen, K., Quidé, Y., Fullerton, J.M., Green, M.J., 2018. Glucocorticoid receptor gene (NR3C1) DNA methylation in association with trauma, psychopathology, transcript expression, or genotypic variation: A systematic review. Neuroscience \& Biobehavioral Reviews.

Wechsler, D., 1999. Wechsler Abbreviated Scale of Intelligence (WASI). The Psychological Corporation, New York, NY.

Wechsler, D., 2001. Wechsler Test of Adult Reading (WTAR). The Psychological Corporation, New York, NY.

World Health Organisation, 2004. ICD-10 : international statistical classification of diseases and related health problems / World Health Organization. World Health Organization, Geneva.

Xin, Y., Chanrion, B., Liu, M.-M., Galfalvy, H., Costa, R., Ilievski, B., Rosoklija, G., Arango, V., Dwork, A.J., Mann, J.J., Tycko, B., Haghighi, F., 2010. Genome-Wide Divergence of DNA Methylation Marks in Cerebral and Cerebellar Cortices. PLOS ONE 5(6), e11357.

Yoshino, Y., Kawabe, K., Mori, T., Mori, Y., Yamazaki, K., Numata, S., Nakata, S., Yoshida, T., Iga, J.-i., Ohmori, T., Ueno, S.-i., 2016. Low methylation rates of dopamine receptor D2 gene promoter sites in Japanese schizophrenia subjects. The World Journal of Biological Psychiatry 17(6), 449-456.

Zeilinger, S., Kühnel, B., Klopp, N., Baurecht, H., Kleinschmidt, A., Gieger, C., Weidinger, S., Lattka, E., Adamski, J., Peters, A., Strauch, K., Waldenberger, M., Illig, T., 2013. Tobacco Smoking Leads to Extensive Genome-Wide Changes in DNA Methylation. PLOS ONE 8(5), e63812. 
bioRxiv preprint doi: https://doi.org/10.1101/607309; this version posted April 12, 2019. The copyright holder for this preprint (which was

not certified by peer review) is the author/funder. All rights reserved. No reuse allowed without permission.

Poly-methylomic Profile Scores for schizophrenia

\section{Figure/Table Legends}

\section{Figure 1}

Title: Figure 1. Group comparisons on raw PMPS score

Legend: Boxplots showing group differences on the PMPS calculated at the most stringent $p$ value threshold ( $\mathrm{p} 1 \times 10^{-7}$ ) between: A) schizophrenia cases and healthy controls, B) bipolar disorder cases and healthy controls, $\mathrm{C}$ ) combined clinical cases (SZ+BD) and healthy controls, and D) bipolar disorder and schizophrenia.

\section{Table 1}

Title: Table 1. Sample demographics

Legend: Demographics statistics for each group (schizophrenia, bipolar disorder, and healthy controls)

\section{Table 2}

Title: Table 2. PMPSs and clinical groups

Legend: Results of logistic regression analyses comparing various groups on each PMPS.

\section{Table 3}

Title: Table 3. PMPSs and SAPS/SANS subscale scores

Legend: Results for regression models examining association between PMPS ( $\left.\mathrm{p}<1 \times 10^{-7}\right)$ and SANS/SAPS global subscales.

\section{Table 4}

Title: Table 4. PMPSs and Medication

Legend: Results from linear regression analyses investigating relationship between PMPS (1 $\mathrm{x} 10^{-7}$ ) for $\mathrm{SZ}$ and medication use in cases. 
Table 1. Sample demographics

\begin{tabular}{|l|l|l|l|l|}
\hline Variable & HC & BD & SZ & Group difference statistics \\
\hline $\mathrm{N}(\%$ Male) & $55(49.09)$ & $59(28.81)$ & $57(61.40)$ & $X^{2}=12.661, p=0.002$ \\
\hline Mean Age in years (SD) & $36.32(11.78)$ & $37.66(12.56)$ & $43.02(11.12)$ & $F(2,168)=5.061, p=0.007, \eta_{p}{ }^{2}=0.057$ \\
\hline Mean Education in years (SD) & $16.84(2.46)$ & $15.68(2.79)$ & $14.23(2.49)$ & $F(2,168)=14.289, p=1.858 \times 10^{-}, \eta_{p}{ }^{2}=0.145$ \\
\hline Mean WTAR (SD) & $110.62(10.30)$ & $105.02(14.78)$ & $100.21(14.43)$ & $F(2,167)=8.495, p=3.066 \times 10^{-4}, \eta_{p}{ }^{2}=0.092$ \\
\hline Mean WASI (SD) & $117.74(12)$ & $114.82(11.39)$ & $107.7(14.077)$ & $F(2,124)=7.121, p=0.001, \eta_{p}{ }^{2}=0.103$ \\
\hline Mean SAPS (SD) & NA & $6.75(8.19)$ & $17.75(14.61)$ & $F(1,111)=24.469, p=2.706 \times 10^{-}, \eta_{p}{ }^{2}=0.181$ \\
\hline Mean SANS (SD) & NA & $15.3(15.83)$ & $29.84(17.26)$ & $F(1,110)=21.605, p=9.361 \times 10^{-}, \eta_{p}{ }^{2}=0.164$ \\
\hline Mean Age of illness onset & NA & $23.42(9.70)$ & $25.04(8.51)$ & $F(1,105)=0.831, p=0.364, \eta_{p}{ }^{2}=0.008$ \\
\hline Mood stabiliser usage (\%; BD vs. SZ) & NA & $45(76.27)$ & $12(21.05)$ & $X^{2}=33.195, p=8.34 \times 10^{-}$ \\
\hline Mean Imiprimine-equivalent dosage (SD) & NA & $26.06(60.05)$ & $63.69(121.99)$ & $F(1,114)=4.491, p=0.036, \eta_{p}{ }^{2}=0.038$ \\
\hline Mean Chlorpromazine-equivalent dosage (SD) & NA & $194(571.09)$ & $476.9(886.43)$ & $F(1,114)=4.204, p=0.043, \eta_{p}{ }^{2}=0.036$ \\
\hline
\end{tabular}

$\mathrm{HC}=$ healthy controls; $\mathrm{BD}=$ bipolar disorder; $\mathrm{SZ}=$ schizophrenia; WTAR=Wechsler Test of Adult Reading; WASI=Wechsler Abbreviated Scale

of Intelligence; SAPS= Scale for Assessment of Positive Symptoms; SANS=Scale for Assessment of Negative Symptoms; SD=standard

deviation 
Table 2. Associations between each of the five PMPS scores and clinical groups

\begin{tabular}{|c|c|c|c|c|c|c|c|c|}
\hline Group comparison & PMPS p-value threshold & \# $C p G s$ & $B$ & OR & CI 95\% & $p$ & FDR q value & Pseudo $\mathbf{R}^{2}$ \\
\hline \multirow{5}{*}{ SZ vs. HC } & 1e-07 (discovery and replication) & 14 & 1.263 & 3.538 & $1.227-10.197$ & 0.018 & 0.029 & 0.5958 \\
\hline & 1e-05 (discovery and replication) & 210 & 1.127 & 3.086 & $1.166-8.166$ & 0.021 & 0.029 & 0.5934 \\
\hline & 0.001 (replication) & 359 & 0.975 & 2.651 & $1.091-6.441$ & 0.029 & 0.029 & 0.5886 \\
\hline & 0.05 (replication) & 684 & 0.923 & 2.516 & $1.126-5.622$ & 0.022 & 0.029 & 0.5919 \\
\hline & 0.5 (replication) & 1051 & 0.803 & 2.233 & $1.080-4.617$ & 0.028 & 0.029 & 0.5883 \\
\hline \multirow{5}{*}{$\mathrm{BD}$ vs. $\mathrm{HC}$} & 1e-07 (discovery and replication) & 14 & -0.077 & 0.926 & $0.423-2.024$ & 0.845 & 0.979 & 0.3998 \\
\hline & 1e-05 (discovery and replication) & 210 & -0.010 & 0.990 & $0.472-2.078$ & 0.979 & 0.979 & 0.3995 \\
\hline & 0.001 (replication) & 359 & -0.130 & 0.878 & $0.424-1.817$ & 0.722 & 0.979 & 0.4006 \\
\hline & 0.05 (replication) & 684 & -0.273 & 0.761 & $0.379-1.530$ & 0.437 & 0.979 & 0.4045 \\
\hline & 0.5 (replication) & 1051 & -0.281 & 0.755 & $0.381-1.495$ & 0.414 & 0.979 & 0.4051 \\
\hline \multirow{5}{*}{ Case vs. Control } & 1e-07 (discovery and replication) & 14 & 0.472 & 1.603 & $0.875-2.935$ & 0.123 & 0.238 & 0.3246 \\
\hline & 1e-05 (discovery and replication) & 210 & 0.465 & 1.592 & $0.911-2.783$ & 0.100 & 0.238 & 0.3269 \\
\hline & 0.001 (replication) & 359 & 0.381 & 1.464 & $0.860-2.494$ & 0.157 & 0.238 & 0.3223 \\
\hline & 0.05 (replication) & 684 & 0.304 & 1.355 & $0.827-2.222$ & 0.224 & 0.238 & 0.3188 \\
\hline & 0.5 (replication) & 1051 & 0.284 & 1.328 & $0.826-2.137$ & 0.238 & 0.238 & 0.3183 \\
\hline \multirow{5}{*}{ SZ vs. BD } & 1e-07 (discovery and replication) & 14 & 0.852 & 2.345 & $0.970-5.666$ & 0.055 & 0.055 & 0.5179 \\
\hline & 1e-05 (discovery and replication) & 210 & 0.834 & 2.303 & $1.035-5.121$ & 0.038 & 0.055 & 0.5232 \\
\hline & 0.001 (replication) & 359 & 0.766 & 2.151 & $1.021-4.532$ & 0.041 & 0.055 & 0.5219 \\
\hline & 0.05 (replication) & 684 & 0.789 & 2.202 & $1.094-4.433$ & 0.025 & 0.055 & 0.5292 \\
\hline & 0.5 (replication) & 1051 & 0.655 & 1.925 & $0.998-3.714$ & 0.048 & 0.055 & 0.5198 \\
\hline
\end{tabular}


Figure 1. Group comparisons on raw PMPS score

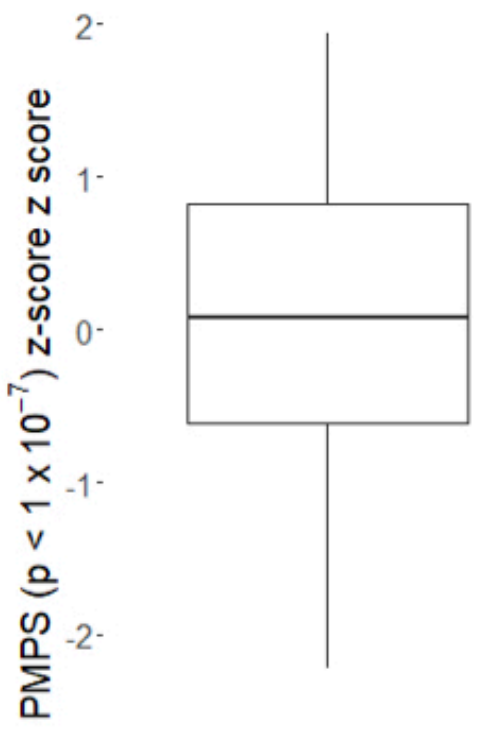

$-3-$

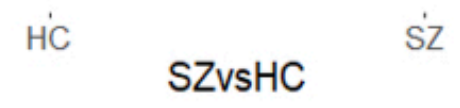

A

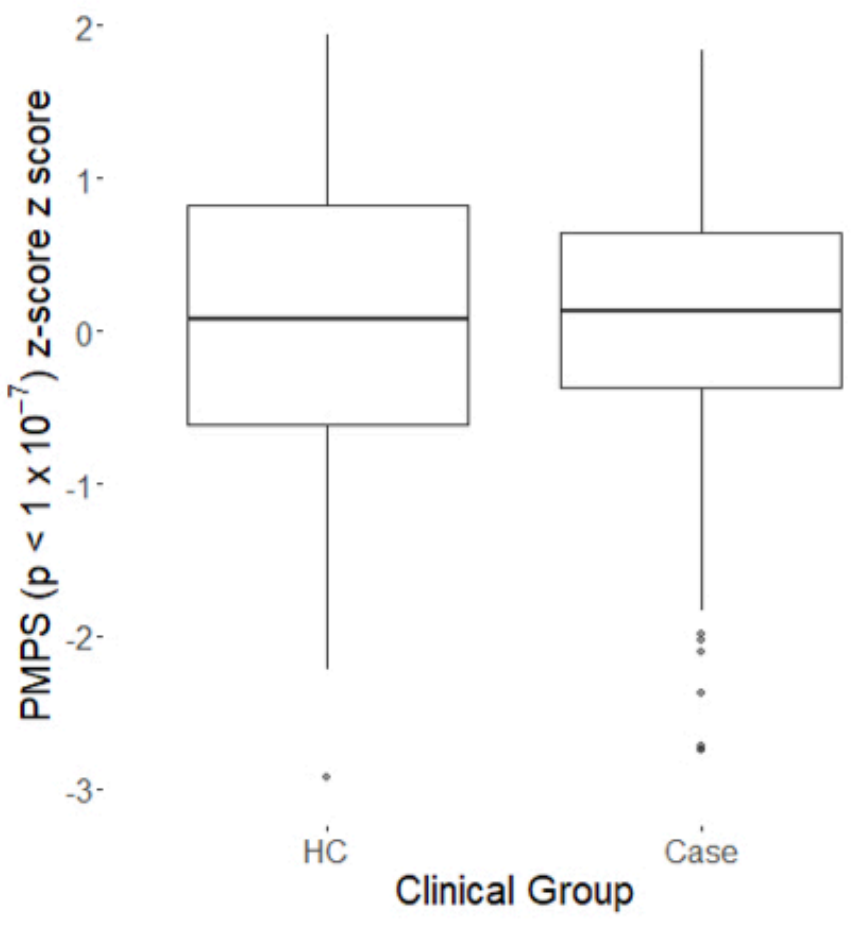

C

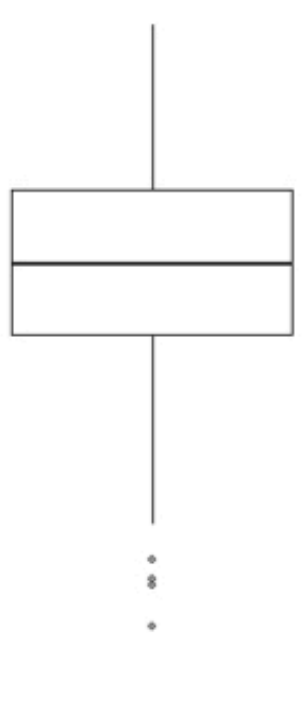

s'z

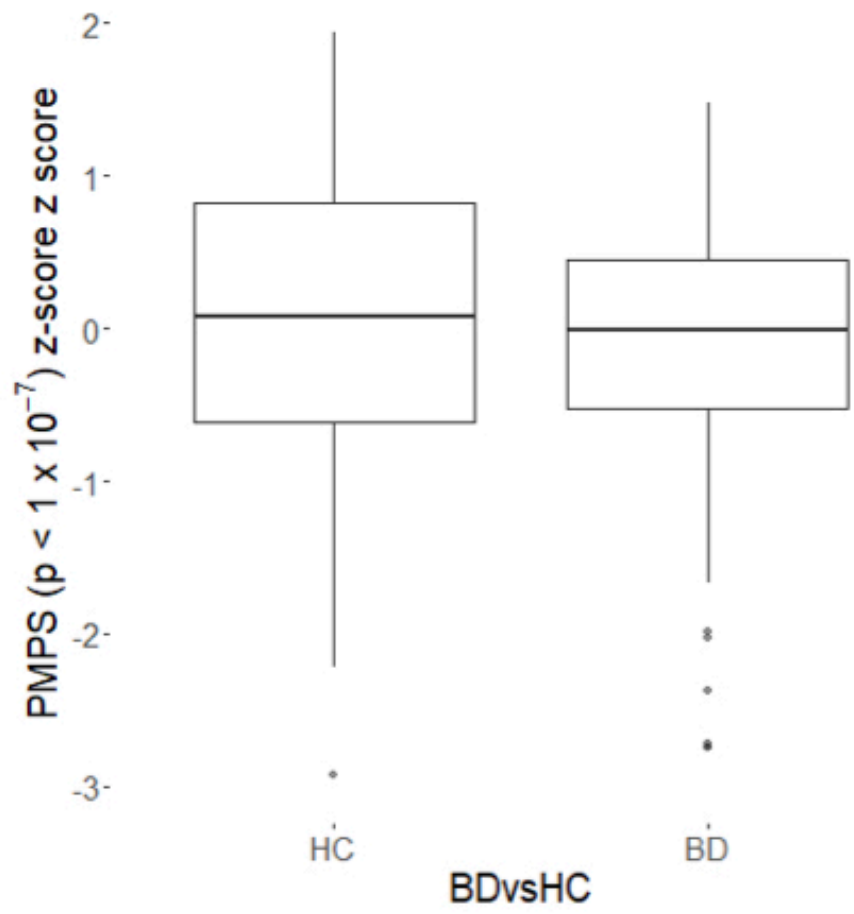

B

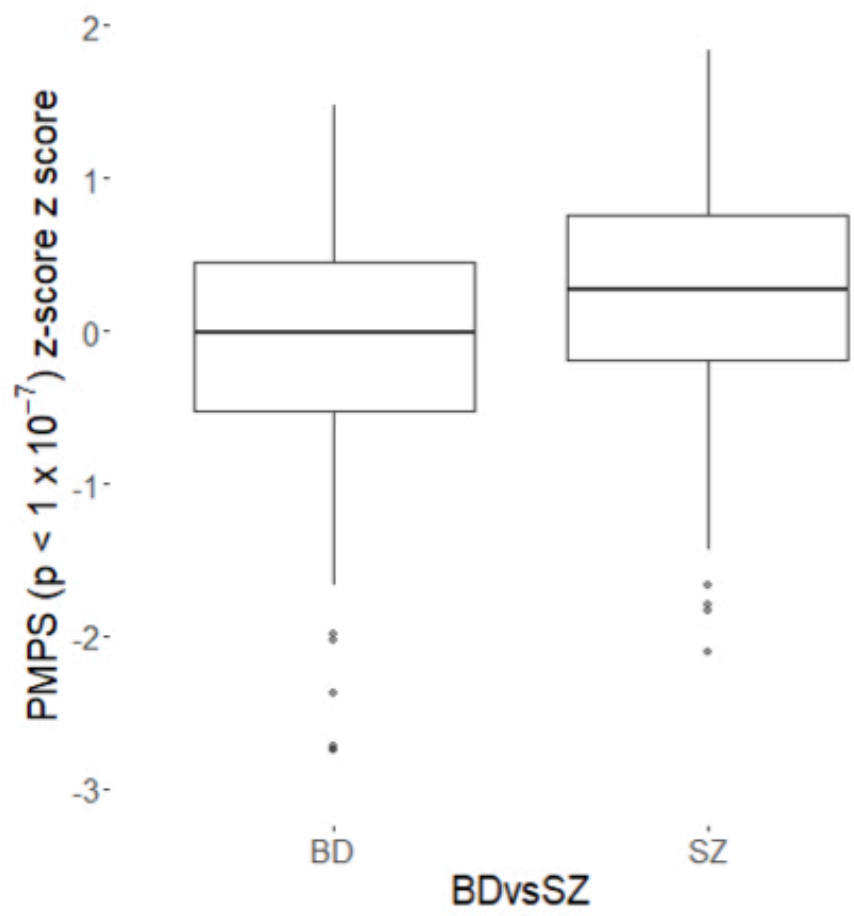

$\mathrm{D}$

Boxplots showing group differences on the PMPS calculated at the most stringent $p$-value threshold ( $1 \times 10^{-7}$ ) between: A) schizophrenia cases and healthy controls, B) bipolar disorder cases and healthy controls, C) combined clinical cases (SZ+BD) and healthy controls, and D) bipolar disorder and schizophrenia. 
Table 3. PMPSs and SAPS/SANS subscale scores

\begin{tabular}{|l|r|r|l|r|}
\hline SANS/SAPS subscale score & \multicolumn{1}{l|}{$\boldsymbol{B}$} & $\boldsymbol{O} \boldsymbol{R}$ & $\mathbf{9 5 \%} \boldsymbol{C I}$ & $\boldsymbol{p}$ \\
\hline SANS Affective Flattening & 0.117 & 1.124 & $0.933-1.354$ & 0.216 \\
\hline SANS Alogia & -0.047 & 0.954 & $0.797-1.142$ & 0.607 \\
\hline SANS Avolition & 0.056 & 1.057 & $0.900-1.242$ & 0.491 \\
\hline SANS Asociality & 0.029 & 1.030 & $0.880-1.204$ & 0.712 \\
\hline SANS Attention & -0.083 & 0.920 & $0.784-1.079$ & 0.302 \\
\hline SAPS Hallucinations & 0.033 & 1.034 & $0.874-1.222$ & 0.696 \\
\hline SAPS Delusions & 0.114 & 1.121 & $0.918-1.369$ & 0.258 \\
\hline SAPS Bizarre behaviour & 0.004 & 1.004 & $0.800-1.259$ & 0.975 \\
\hline SAPS Thought disorder & -0.055 & 0.946 & $0.827-1.082$ & 0.414 \\
\hline
\end{tabular}


Table 4. Results from linear regression analyses investigating relationship between PMPS $\left(1 \times \mathbf{1 0}^{-7}\right)$ for $\mathrm{SZ}$ and medication use in cases.

\begin{tabular}{|l|r|r|l|l|}
\hline Cohort and Medication & \multicolumn{1}{|l|}{$B$} & OR & $95 \% C I$ & $p$ \\
\hline SZ & -0.001 & 0.999 & $0.996-1.002$ & 0.378 \\
\hline Imipramine Equivalent Dosage & 0.000 & 1.000 & $1.000-1.001$ & 0.115 \\
\hline Chlorpromazine Equivalent Dosage & 0.217 & 1.242 & $0.482-3.198$ & 0.641 \\
\hline Mood Stabiliser Usage & 0.003 & 1.003 & $0.996-1.009$ & 0.419 \\
\hline BD & 0.000 & 1.001 & $0.999-1.001$ & 0.807 \\
\hline Imipramine Equivalent Dosage & -0.055 & 0.946 & $0.485-1.848$ & 0.867 \\
\hline Chlorpromazine Equivalent Dosage & 0.001 & 1.001 & $0.999-1.002$ & 0.460 \\
\hline Mood Stabiliser Usage & 0.000 & 1.000 & $1.000-1.000$ & 0.332 \\
\hline Combined psychosis group (SZ+BD) & -0.202 & 0.817 & $0.586-1.138$ & 0.228 \\
\hline Imipramine Equivalent Dosage & & & \\
\hline Chlorpromazine Equivalent Dosage &
\end{tabular}

Note: The optimal PMPS score for SZ was based on the top $14 \mathrm{CpG}$ sites associated with $\mathrm{SZ}$ as reported by Hannon et al. ${ }^{5}$ 